\title{
Beitrag zur Prozessstandardisierung in der Intralogistik
}

\author{
DiPL.-ING. O. FIGGENER \\ PROF. DR. M. TEN HOMPEL \\ FRAUNHOFER-INSTITUT FÜR MATERIALFLUSS UND LOGISTIK (IML), DORTMUND
}

\section{Zusammenfassung}

Ein dynamisches Umfeld erforderte die permanente Anpassung (intra-)logistischer Prozesse zur Aufrechterhaltung der Leistungs- und Wettbewerbsfähigkeit von Unternehmen. In der Standardisierung von Prozessen und in unternehmensübergreifenden Prozessmodellen wurden Schlüsselfaktoren für ein effizientes Geschäftsprozessmanagement gesehen, insbesondere in Netzwerken. In der Praxis fehlten wissenschaftlich fundierte und detaillierte Referenzprozessmodelle für die (Intra-)Logistik. Mit der Erforschung und Entwicklung eines Referenzprozessmodells und der prototypischen Realisierung einer Prozess-Workbench zur generischen Erstellung wandelbarer Prozessketten wurde ein Beitrag zur Prozessstandardisierung in der Logistik geleistet. Im Folgenden wird der beschrittene Lösungsweg dargestellt, der erstens aus der Entwicklung eines Metamodells für die Referenzmodellierung, zweitens aus dem empirischen Nachweis der Generierung eines Referenzprozessmodells aus „atomaren“ Elementen und drittens aus der Modellevaluation bestand.

\begin{abstract}
To sustain performance and competitiveness of enterprises in a dynamic environment a permanent adaptation of (intra-)logistic processes was required. Standardization of processes and corporate overlapping process models were considered as key factors for an efficient business process management, particularly in networks. In Practice there were no scientific established and detailed reference process models for the intra logistic sector. With the research and development of a reference process model and the prototypic implementation of a process workbench for the generic creation of changeable process chains a contribution to process standardization in logistics was made. Below the chosen solution process is described, which consisted first in the development of a metamodel for the reference modelling, second in the empiric confirmation of the generation of a reference process model from "atomic" elements and third in the model evaluation.
\end{abstract}

\section{Einleitung}

\subsection{Motivation}

Geopolitische Veränderungen, volkswirtschaftliche Neuorientierung und technologische Innovationen führten in Summe zu einem ständigen Wandel des unternehmerischen Umfelds. Die Unternehmensentwicklung konnte zu einem Unternehmenswachstum, einer Integration neuer Unternehmensbestandteile, einer Nutzung von Standortvorteilen oder einem Outsourcing von Funktionsbereichen führen. Die Intralogistik musste diesen Änderungen angepasst werden, um leistungsfähig zu bleiben [Figg06, S. 26-29]. Der Logistik fiel bei der Unternehmensentwicklung eine wichtige Rolle zu, weil sie für die Planung, Steuerung und Überwachung der Beschaffung, Produktion, Distribution und Entsorgung verantwortlich war. Zentrale Elemente der Logistik waren Läger, die die Bindeglieder in logistischen Netzen darstellten. Die Intralogistik als Teildisziplin der Logistik „,beschreibt den innerbetrieblichen Materialfluss, der zwischen den unterschiedlichsten ,Logistikknoten’ stattfindet [...]“ [tHHe06, S. 105]. Die Komplexität eines Lagers und das Zusammenspiel mit seiner Umwelt erforderten ein effizientes Management mit dem Ziel einer hohen Zuverlässigkeit und Leistungsfähigkeit der Intralogistik. Innovative (Informations-) Techniken wie standardisierte Warehouse Management-Systeme oder die Radio Frequency Identification unterstützten die operativen Lagerprozesse und stellten einen wesentlichen Beitrag zur Leistungsfähigkeit der Logistik dar.

Die isolierte Betrachtung eines Unternehmens war vor dem Hintergrund einer langfristig erfolgsorientierten Unternehmensentwicklung nicht zielführend. So wurden und werden beispielsweise im Sonderforschungsbereich 559 an der Universität Dortmund große Netze der Logistik analysiert und Methoden zu deren Modellierung und Beherrschung erforscht. Ziel des Transferprojekts „Modellierung eines Virtuellen Lagers“ war und ist die Erforschung einer dynamischen Kooperationsform eigenständiger Unternehmen, bei der räumlich und organisatorisch getrennte Lager- und Transportkapazitäten zu einem Lagersystem mit zentraler Disposition zu- 
sammengefasst werden. Diese Form der Zusammenarbeit wird ,Virtuell' genannt, da es sich nicht um ein Lager mit einem physischen Standort handelt, sondern um einen dynamischen Lagerverbund, dessen Standorte sich mit den kooperierenden Unternehmen ändern. Den Anbietern logistischer Kapazitäten stehen Nutzer gegenüber. Sie verwenden die vom Lagerbetreiber zur Verfügung gestellten Flächen temporär [SFB06]. Die Erforschung eines dezentralen, selbstorganisierenden Materialflusses war und ist ein erklärtes Ziel des Transferprojekts. Echtzeitnahe Standortinformationen und zuverlässige Auskünfte über die Prozessfähigkeit der logistischen Ressourcen waren hierzu die entscheidenden Informationen. Die Prozesskompetenz der Netzwerkpartizipatoren sollte durch die Anerkennung von zu erforschenden Referenzprozessen nachgewiesen werden.

Die sich ändernden Anforderungen an Läger und technische Entwicklungen führten häufig zu Prozessvarianten und zu einer Prozessvielfalt für vergleichbare Abläufe im Lager, deren Notwendigkeit selten konsequent in Frage gestellt wurde. Das Management von Geschäftsprozessen rückte zunehmend in den Fokus der Unternehmensführung, dies galt auch für die Logistik [Wild01, S. 7]. Aufgabe des Geschäftsprozessmanagements war die Steuerung und Gestaltung von Abläufen.

Für Prozesse, die keine Alleinstellungsmerkmale oder Wettbewerbsvorteile beinhalten, sollte eine Ausrichtung an einer geeigneten Referenz möglich sein. Becker/Schütte trafen eine eindeutige Einschätzung des Nutzens von Referenzmodellen. Sie wirkten ihnen zufolge kostenminimierend, erlösmaximierend und risikominimierend [BeSc04, S. 80ff.]. Die Kostenminimierung resultierte aus einer vereinfachten Identifikation von Prozessen, die unternehmensindividuell zu gestalten sind. Der unternehmensbezogene Modellentstehungsprozess wurde somit beschleunigt. Fettke/Loos untersuchten systematisch verbreitete Referenzmodelle [FeLo04, S. 16]. Die aufgeführten Referenzmodelle für die Logistik zeichneten sich durch

- $\quad$ einen geringen Detaillierungsgrad,

- $\quad$ einen bedingt hierarchischen Aufbau,

- das Fehlen einer detaillierten, branchenbezogenen Prozessbibliothek und

- das Fehlen einer generischen Erstellung von Prozessketten

aus. Sie waren als Referenzprozessmodelle (RPM) für die Prozessstandardisierung nur bedingt geeignet.

\subsection{Zielsetzung}

Ein zu beobachtender Trend in der Industrie war die Standardisierung planerischer, steuernder und operativer Prozesse. Eine Studie zur Zukunft der volkswirtschaftlich gewichtigen Automobilindustrie unterstrich die Bedeutung abgestimmter unternehmensübergreifender Prozessmodelle [VDA04]. Am Beispiel der Automobilindustrie wurde auch deutlich, dass das Management der externen, nicht OEM ${ }^{1}$-eigenen Wertschöpfung in Quantität und Komplexität in erheblichem Maße zunehmen würde [VDA04, S. 40ff.]. Die Qualität der Zusammenarbeit in dem entstehenden Netzwerk würde unter anderem von einem gemeinsamen Prozessmodell getragen, so das Fazit.

Die Untersuchung unternehmensspezifischer Logistikprozesse dreier OEM der Automobilindustrie ergab eine Prozessübereinstimmung in der Domäne „Automotive Ersatzteillogistik“ von nur 56 \%, Aussagen über die Vollständigkeit eines logistischen Prozessmodells waren aufgrund eines fehlenden wissenschaftlich fundierten Ordnungsrahmens nicht möglich. Verfügbare logistische Referenz(prozess-)modelle waren aufgrund ihres Abstraktionsgrades jedoch nur bedingt für die Prozessgestaltung geeignet. Eine Ausrichtung oder Vereinheitlichung von Prozessen, beispielsweise an RPM, kann als „Prozessstandardisierung“ bezeichnet werden. Ziel war - und ist - die Definition und Gestaltung von einheitlichen Standardprozessen, die für vergleichbare Abläufe im Unternehmen stehen. Derartige Standardprozesse würden den Planungs- und Steuerungsaufwand reduzieren und somit zur Komplexitätsreduzierung in logistischen Systemen beitragen. Gleichzeitig müssten sie effizient an geänderte Randbedingungen angepasst werden können.

Aus dem dargestellten Status quo wurde folgender Forschungsbedarf abgeleitet: Die systematische Entwicklung eines vollständigen, wandelbaren und domänenspezifischen Referenzprozessmodells für die Intralogistik als Basis für die Prozessstandardisierung.

Die Arbeit verfolgte zwei Zielsetzungen: Ein Ziel war die Entwicklung eines Metamodells, das den Anforderungen des Geschäftsprozessmanagements ebenso genügt wie den Erkenntnissen der Referenzmodellierungsforschung. Es sollte den systematischen Übergang von implizitem Prozesswissen in explizites ermöglichen. Ein zweites Ziel lag im empirischen Nachweis, ein Referenzprozessmodell auf der Basis ,atomarer“ Prozesselemente entwickeln zu können. Der Forderung nach Wandelbarkeit wurde mit der Erforschung und Definition ,,atomarer“ Prozesselemente entgegen getreten, die eine generische Erstellung wandelbarer Prozessketten ermöglichten. Mit der Konzeption und der prototypischen Realisierung einer ProzessWorkbench sollte dieser Nachweis erbracht werden.

\footnotetext{
${ }^{1}$ OEM: Original Equipment Manufacturer
} 


\section{Lösungsweg}

\subsection{Metamodell}

\subsubsection{Phasen des Metamodells}

Für die Erstellung eines idealtypischen Abbildes unternehmensspezifischer Prozesse, eines RPM, war es nicht ausreichend, die Abläufe eines einzigen Unternehmens zu analysieren. Der Grad der Überdeckung zwischen der Realität und den idealtypischen (Referenz-) Prozessen stieg naturgemäß mit der Anzahl Unternehmen, die in die Modellbildung einbezogen wurden. Ausgangspunkt für die Erstellung eines RPM waren die Unternehmensprozesse dreier OEM der Automobilindustrie. Sie wurden mittels eines Metamodells in ein hierarchisches Referenzmodell überführt. Die „Grundsätze ordnungsmäßiger Modellierung“ [Schü98] waren als wissenschaftlich anerkannte Methode die Basis zur Modellerstellung. Für die systematisch reproduzierbare Referenzmodellierung und zur Sicherstellung der Modellqualität wurde ein sechsphasiges Metamodell entwickelt (siehe Tab.1).

Tabelle 1: Sechs Phasen des Vorgehensmodells zur Erstellung von RPM

\begin{tabular}{lllll}
\hline Phase & Inhalt & Ergebnis & Vorgehensweise & \\
\hline 1.1 & $\begin{array}{l}\text { Interpretation und Umsetzung } \\
\text { der Grundsätze ordnungs- } \\
\text { mäßiger Modellierung }\end{array}$ & $\begin{array}{l}\text { Syntax und Semantik, } \\
\text { Prozess-Workbench }\end{array}$ & $\begin{array}{l}\text { modell- } \\
\text { übergreifend }\end{array}$ & deduktiv \\
\hline 1.2 & $\begin{array}{l}\text { Entwicklung eines } \\
\text { Ordnungsrahmens }\end{array}$ & $\begin{array}{l}\text { Grenzen der } \\
\text { Anwendungsdomäne }\end{array}$ & $\begin{array}{l}\text { modell- } \\
\text { übergreifend }\end{array}$ & deduktiv \\
\hline 2.1 & $\begin{array}{l}\text { Ermittlung der signifikanten } \\
\text { Merkmale einer Branche be- }\end{array}$ & Branchencharakteristika & $\begin{array}{l}\text { domänen- } \\
\text { spezifisch }\end{array}$ & induktiv \\
& zogen auf die Domäne des RPM & & \\
\hline 2.2 & Abstraktion der Prozesse in der & $\begin{array}{l}\text { Datenbasis für die Model- } \\
\text { lierung (Prozesswissen) }\end{array}$ & $\begin{array}{l}\text { domänen- } \\
\text { spezifisch }\end{array}$ & induktiv \\
\hline 2.3 & Domäne & $\begin{array}{l}\text { Referenzielle Prozesse } \\
\text { einer Domäne }\end{array}$ & $\begin{array}{l}\text { domänen- } \\
\text { spezifisch }\end{array}$ & induktiv \\
& Vergleichende Prozessanalyse & Branchenspezifisches & $\begin{array}{l}\text { domänen- } \\
\text { spezifisch }\end{array}$ & induktiv \\
\hline 2.4 & Modellierung der & Referenzprozessmodell & & \\
& Referenzprozesse & &
\end{tabular}

Die Interpretation der Grundsätze ordnungsmäßiger Modellierung (Phase 1.1) hatte grundlegende Wirkung auf alle nach dem Metamodell erstellten RPM. So führte der Grundsatz der Sprachadäquanz zu den Hierarchiestufen des Modells, die Darstellungsart der Prozesse und den Sprachgebrauch. Für die Verwaltung der Prozesse wurde eine Prozess-Workbench prototypisch entwickelt. Einschlägige Prozesse im Lager bildeten den modellübergreifenden Ordnungsrahmen für die RPM (Phase 1.2). Diese deduktiven Elemente stellten bildlich gesprochen die Basis des Metamodells dar. Die Identifizierung der Merkmale einer Branche erfolgte domänenspezifisch (Phase 2.1). Ihre Berücksichtigung bei der Referenzmodellierung bewirkte, dass die Prozesse auch den unternehmensübergreifenden Charakteristika einer Branche genügten. Das unternehmensspezifische Prozesswissen wurde in geeigneter Weise abstrahiert (Phase 2.2). Der Detaillierungsgrad der Prozesse musste mindestens dem des Ordnungsrahmens entsprechen, damit das RPM eine vergleichbare Aussagekraft hatte. Um sicherzugehen, dass das RPM alle relevanten Lagerprozesse unabhängig vom Vorkommen in den betrachteten Unternehmen beinhalteten, erfolgten eine vergleichende Prozessanalyse und eine Ergänzung der abstrahierten, unternehmensspezifischen Prozesse um einschlägige Lagerprozesse (in der Phase 2.3). Abschließend wurde unter Verwendung der Modellierungssprache ein RPM erstellt. Diese induktiven Elemente sind in sich geschlossen und stellten bildlich einen Kreis dar. 


\subsubsection{Prozess-Workbench}

Zur Anpassung der Referenzprozesse wurden eine effiziente Methode und ein Tool benötigt, die den Änderungsaufwand auf ein Minimum reduzierten. Die Lösung dieser Problemstellung lag in der Entwicklung einer ProzessWorkbench, die Prozessketten aus „atomaren“ Prozesselementen generierte. Ein derartiges Tool war nicht vorhanden. Die Prozess-Workbench wurde als Arbeitskonsole zur Verwaltung von Prozesswissen konzipiert, die sowohl die Speicherung der modellierten Prozessketten und den daraus abgeleiteten Referenzprozessen als auch die Verwaltung unternehmensspezifischer Standardprozesse erlaubte. Sie wurde modular mit den drei Elementen

- „Graphics“,

- „Engine“ und

- „Repository“

aufgebaut.

Die folgende Abbildung verdeutlicht die Struktur der Prozess-Workbench:

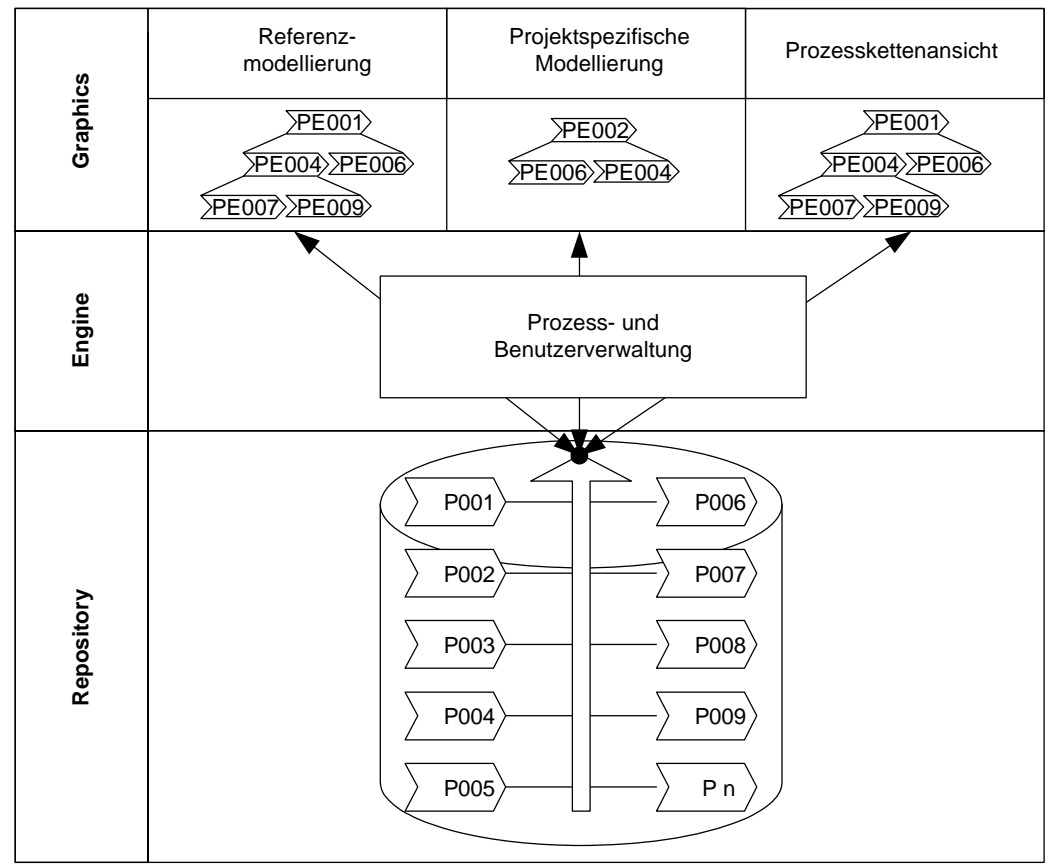

Abbildung 1: Modularer Aufbau der Prozess-Workbench

Die Modellierung der Prozesse erfolgte gemäß dem Prozesskettenparadigma des SFB 559. Auf seiner Basis wurden in dem Modul „Graphics“ die Prozessketten visualisiert. Es ermöglichte die Anzeige, die Bearbeitung oder die Neugestaltung von Prozessketten. Die Prozessketten setzten sich vollständig generisch aus Prozesselementen zusammen. Diese wurden in dem „Repository“ gespeichert. Bei einer Änderung von Prozessen waren nur einzelne Prozesselemente zu löschen, zu verändern oder zu ergänzen. Für die Nutzung der Workbench stand mit der „Engine“ ein Verwaltungsmodul zur Verfügung, das den Zugriff auf die gespeicherten Prozesse ermöglichte. Dieses Konzept ermöglichte die dynamische Anpassung einzelner Prozessmodule/-elemente, ohne die gesamte Prozesskette überarbeiten zu müssen. Die geforderte wirtschaftliche Erweiterung referenzieller und standardisierter Prozesse wurde auf diese Weise rationell möglich.

\subsubsection{Ordnungsrahmen und Detaillierungsgrad}

Trotz unterschiedlicher Ausprägungen einzelner Läger ließen sich Funktionsbereiche identifizieren, die standardmäßig vertreten waren. Einschlägige Logistikprozesse waren aus wissenschaftlichen und praxisnahen Beschreibungen bekannt [tHoS03] [VDI04]. Aus ihnen wurde ein prozessorientierter Ordnungsrahmen für ein logistisches Referenzmodell abgeleitet. Ziel des Ordnungsrahmens war es, die charakteristischen Elemente eines ,Originals' zu gliedern und in Beziehung zueinander zu setzen. Bei der Referenzmodellierung musste sichergestellt werden, dass die Prozesse des Ordnungsrahmens von dem Modell abgedeckt wurden. Aus den einschlägig beschriebenen Logistikprozessen wurde der tabellarische Ordnungsrahmen abgeleitet: 
Tabelle 2: Hauptprozesse, Prozessmodule und Prozesselemente einschlägiger Logistikprozesse (Auszug)

\begin{tabular}{|c|c|c|c|}
\hline Nr. & Hauptprozesse & Prozessmodule & Prozesselemente \\
\hline \multirow[t]{8}{*}{1} & Ware annehmen & Verkehrsfläche steuern & Lieferavise abrufen \\
\hline & & & Kapazitäten planen \\
\hline & & & Lieferung anmelden \\
\hline & & & Lieferung unter Vorbehalt buchen \\
\hline & & & Ladetor zuweisen \\
\hline & & & Entladetor ansteuern \\
\hline & & Ware entladen & Lieferung abladen \\
\hline & & & $\ldots$ \\
\hline
\end{tabular}

In der Literatur und in einschlägigen Normen wurden 92 einzelne Logistikfunktionen bzw. -prozesse im Lager unterschieden. Diese konnten thematisch in 30 Prozessmodule eingeteilt werden. „Bottom-up“ erfolgte in der letzten Hierarchiestufe die Bündelung der Module zu 8 Hautprozessen.

Mit der vorgestellten Definition einschlägiger Prozesse für die Lagerlogistik wurden sämtliche Funktionsbereiche abgedeckt, ein Prozessmodell konnte als „vollständig“ bezeichnet werden, wenn es sich in dem Ordnungsrahmen befand. Damit existierte ein quantifizierbarer Maßstab für die Granularität beziehungsweise für den Detaillierungsgrad von Prozessen im Lager, der somit wie folgt definiert wurde:

Der Detaillierungsgrad wird definiert als „Anzahl der Prozesselemente eines Modells“ bezogen auf die „Anzahl der Prozesselemente des Ordnungsrahmens eines Modells“.

Anmerkung:

Notwendige Voraussetzung ist, dass beide Modelle in derselben Domäne Gültigkeit haben. Um den Detaillierungsgrad von RPM auf Basis ereignisgesteuerter Prozessketten zu ermitteln, werden „Ereignisse“ und „Prozesse“ jeweils als Prozesselemente im Sinne der oben genannten Definition betrachtet. Somit kann auch der Detaillierungsrad ereignisgesteuerter Prozessketten bestimmt werden.

Ein Detaillierungsgrad $>1,0$ bezeichnete ein Prozessmodell, das feiner ist als der Ordnungsrahmen, ein Detaillierungsgrad $<1,0$ kennzeichnete ein Prozessmodell, das gröber ist als der Ordnungsrahmen.

\subsection{Fallstudie: Entwicklung eines Referenzprozessmodells}

\subsubsection{Ermittlung signifikanter Merkmale der automobilen Ersatzteillogistik}

Ein typisches Zentrallager in der automobilen Ersatzteillogistik wickelte Sendungen von deutlich über 2.500 Lieferanten ab. Das Gewicht der täglich angelieferten Waren überschritt 1.500 Tonnen, die in 200 bis 300 Lkws angeliefert wurden. Diese Menge verließ in anderer Zusammensetzung täglich das Zentrallager, es wurden zum Teil über 50.000 Auftragspositionen bearbeitet. Die Empfänger dieser Waren waren im Wesentlichen die weltweiten Großhändler, deren Zahl sich je nach Automobilhersteller zwischen 15 und 50 belief. Die Distribution wurde typischerweise durch Gebietsspediteure übernommen. Weiter erfolgte aus den meisten Zentrallägern die Belieferung der regionalen Einzelhändler, ebenfalls durch Gebietsspediteure. Die folgende Tabelle beinhaltet auszugsweise typische Branchenmerkmale, die fett hervorgehoben wurden. 
Tabelle 3: Typische Ausprägungen der Branchenmerkmale „Automotive“

\begin{tabular}{|c|c|c|c|c|c|}
\hline Merkmal & \multicolumn{5}{|c|}{ typische Ausprägungen } \\
\hline $\begin{array}{l}\text { Lagertypen } \\
\text { (Warenver- } \\
\text { teilungsstruktur) }\end{array}$ & Zentrallager & Regionallager & & & \\
\hline Lageranzahl & ein Standort & $\begin{array}{l}\text { mehrere } \\
\text { Standorte }\end{array}$ & & & \\
\hline Bauformen & $\begin{array}{l}\text { geschlossene } \\
\text { Lager }\end{array}$ & $\begin{array}{l}\text { überdachte } \\
\text { Lager }\end{array}$ & Freilager & & \\
\hline Bauhöhe & Flachlager & $\begin{array}{l}\text { mittelhohes } \\
\text { Lager }\end{array}$ & Hochregallager & Lagersilo & \\
\hline $\begin{array}{l}\text { Systematik der } \\
\text { Lagermittel }\end{array}$ & Bodenlagerung & $\begin{array}{l}\text { statische } \\
\text { Regallagerung }\end{array}$ & $\begin{array}{l}\text { dynamische } \\
\text { Regallagerung }\end{array}$ & $\begin{array}{l}\text { Lagerung auf } \\
\text { Fördermitteln }\end{array}$ & \\
\hline $\begin{array}{l}\text { Stellung des } \\
\text { Lagers im logisti- } \\
\text { schen Prozess }\end{array}$ & $\begin{array}{l}\text { Produktions- } \\
\text { lager }\end{array}$ & $\begin{array}{l}\text { Distributions- } \\
\text { lager }\end{array}$ & $\begin{array}{l}\text { Umschlags- } \\
\text { lager }\end{array}$ & Handelslager & werknahes Lager \\
\hline$\cdots$ & $\cdots$ & $\cdots$ & $\cdots$ & & \\
\hline
\end{tabular}

Die Ersatzteillogistik der Automobilhersteller war durch einen dreistufigen Distributionsweg gekennzeichnet. Von einem Zentrallager wurden weltweit die Regionalläger der Großhändler beliefert, die ihrerseits die Händler versorgten. Die Organisationseinheit „Zentrallager“ verteilte sich auf mehrere Standorte. Es handelte sich um geschlossene Läger in mittelhoher Bauform mit integrierten Hochregallägern. Die Lagerung der Ware erfolgte als Bodenlagerung oder statisch bzw. dynamisch in Regalen. Die Zentralläger waren Distributionsläger, eine Produktionsversorgung erfolgte nur in seltenen Ausnahmefällen. Die hier vorgestellten Branchenmerkmale stellen einen Auszug der vollständigen Übersicht dar.

\subsubsection{Vergleichende Prozessanalyse}

Gemäß dem Metamodell wurden die Prozesse identifiziert, die in mehreren Unternehmen vorzufinden waren. Diese wurden auf Plausibilität überprüft, mit den einschlägigen Logistikprozessen des Ordnungsrahmens abgeglichen und ggf. ergänzt. Mit Durchlaufen dieser Arbeitsschritte wurden die Referenzprozesse der Domäne festgelegt. Aufgrund der Komplexität und der Übersichtlichkeit wurden die Referenzprozesse zur Vorbereitung der eigentlichen Prozessmodellierung tabellarisch aufgeführt. Die folgende Tabelle beinhaltet auszugsweise die Hauptprozesse, Prozessmodule und Prozesselemente.

Tabelle 4: Referenzprozesse (Auszug)

\begin{tabular}{lll}
\hline Hauptprozesse & Prozessmodule & Prozesselemente \\
\hline Ware annehmen & Verkehrsfläche steuern & LKW fährt ein \\
& Bahn fährt ein \\
& Einfahrt anmelden \\
& Ankunftszeit erfassen \\
& Entladezeit zuweisen \\
& Entladetor zuweisen \\
& Entladetor anfahren \\
\hline Ware entladen & Einheiten abladen \\
& Lieferung mit Lieferschein vergleichen \\
& Lieferschein quittieren \\
& Lieferung auf Transportschäden prüfen
\end{tabular}


Leergut austauschen

LKW fährt aus

Bahn fährt aus

Abfahrtszeit erfassen

\subsubsection{Visualisierung der Referenzprozesse}

In der letzten Phase des $\Omega$-Metamodells wurden die Prozesse des RPM modelliert. Die einzelnen Lagerbereiche wurden durch Funktionsflächen für den innerbetrieblichen Transport verbunden. Der Materialfluss in die Lagerbereiche und aus den Lagerbereichen erfolgte durch Stapler oder automatische Fördertechnik. Die einzelnen Funktions- und Lagerbereiche wurden über Bereitstellflächen angesteuert. Die Warenübergabe an automatische Lagersysteme erfolgte durch Übergabestationen.

Das folgende typische Zentrallagerlayout dient dem besseren Verständnis des Materialflusses. Es beinhaltet die im Referenzmodell verwendeten Quellen und Senken:

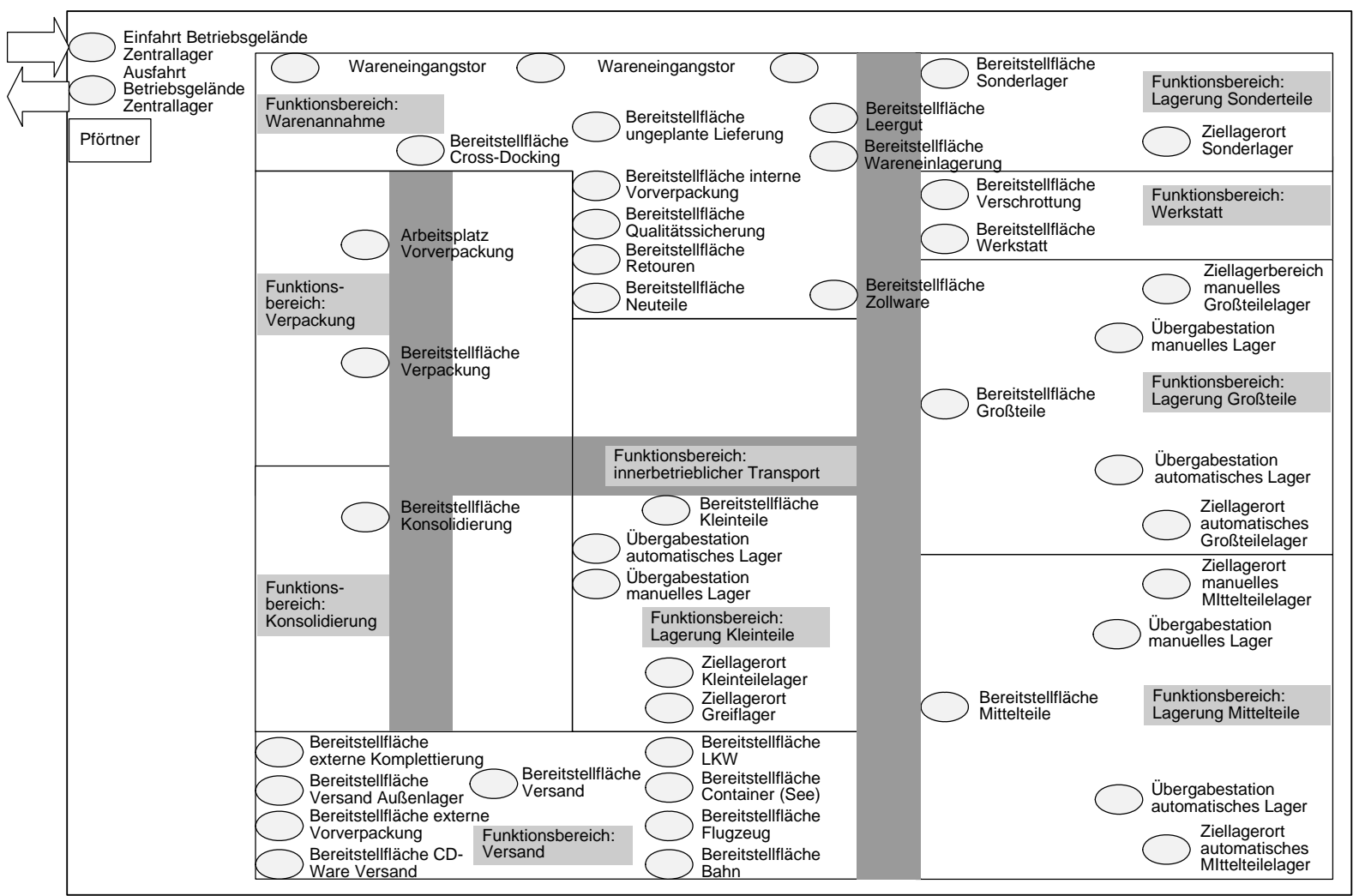

Abbildung 2: Layout des Zentrallagers des RPM

Das RPM orientierte sich an diesem Layout. Die Hauptprozesse

- „Ware annehmen“,

- „Ware einlagern“,

- „Ware auslagern“,

- „Ware verpacken“,

- „Ware konsolidieren“ und

- „Ware versenden“

wurden den Funktionsbereichen zugeordnet. Das $\Omega$-Metamodell sah eine hierarchische Gliederung der Prozesse mit zunehmendem Detaillierungsgrad vor. Die Zusammenhänge der Prozesse im RPM „Automotive Ersatzteillogistik“" wurden an der ablauflogischen Aneinanderreihung der Hauptprozesse verdeutlicht. Die Quellen und Senken des Materialflusses wurden am Beginn und Ende eines Hauptprozesses aufgeführt. Die Prozessmodule der einzelnen Hauptprozesse gaben einen feineren Einblick in die Abläufe. Auch sie begannen in der Material- 
flussebene mit einer Quelle.Die Prozesselemente der einzelnen Prozessmodule beinhalteten einen detaillierten Einblick in die Abläufe. Auch sie begannen in der Materialflussebene mit einer Quelle und endeten stets in einer Senke.

Um das Prinzip der Referenzmodellierung zu erläutern, wird in der folgenden Abbildung das Prozessmodul „Verkehrsfläche steuern“ des Hauptprozesses „Ware annehmen“ auszugsweise vorgestellt:

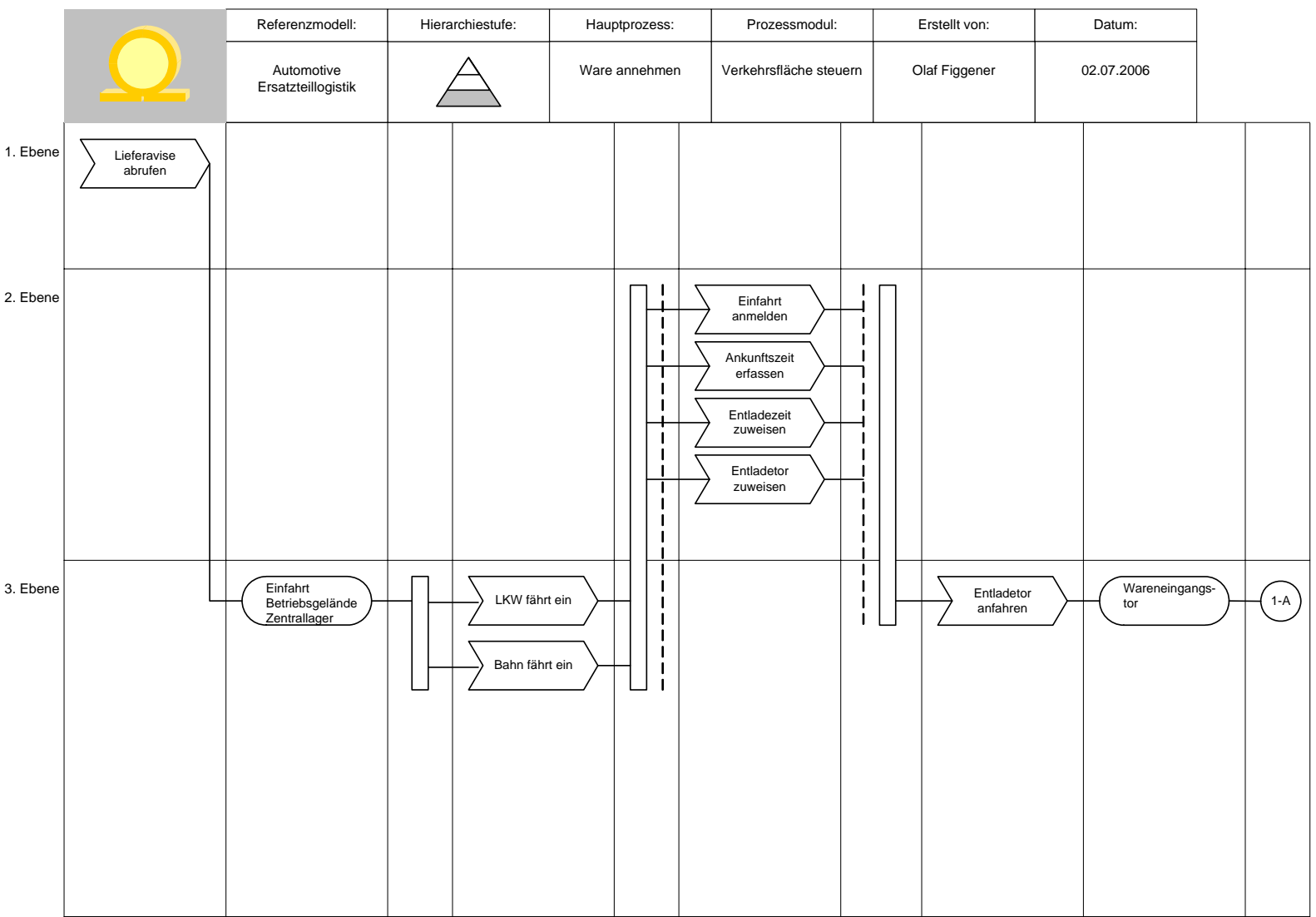

Abbildung 3: Prozesselemente des Prozessmoduls „Verkehrsfläche steuern“

In der ersten Ebene wurde der vorauseilende Informationsfluss beschrieben, in der zweiten der koordinierende. Die Materialflussbeschreibung erfolgte in der dritten Ebene. Im Wareneingang wurden die Weichen des Materialflusses gestellt, dessen Quelle im RPM die Einfahrt zum Betriebsgelände des Zentrallagers war. Die Ware wurde entweder mit einem LKW oder der Bahn angeliefert. Die hohe Anzahl an LKW-Lieferungen erforderte ein exaktes Management des Verkehrshofes, um eine reibungslose Abwicklung zu gewährleisten. Hierzu gehörten zeitgleich die Anmeldung der Lieferung, die Erfassung der Anlieferung und die Zuweisung einer Entladezeit sowie eines Entladetores. Mit dem Anfahren des Entladetores wurde die erste Senke des Materialflusses, das „Wareneingangstor“, erreicht. Der Prozess wurde in dem Prozessmodul „Ware entladen“ fortgesetzt.

\subsection{Evaluation des Referenzprozessmodells}

Das zu evaluierende RPM war ein Wissensspeicher in der Domäne „Automotive Ersatzteillogistik“, die eine Subdomäne der Domäne „Intralogistik“ darstellte. Ein Warehouse Management System (WMS) repräsentierte aus informationstechnischer Sicht ebenfalls einen Ausschnitt der Domäne „Intralogistik“, da die Abläufe in den Funktionsbereichen eines Lagers vielfach durch standardisierte WMS unterstützt wurden [tHoS03, S. 23f.] [Figg05, S. 75]. Ten Hompel belegte 1999 mit dem Objekt-/Ebenenmodell der Lagerverwaltung [tHom99, S. 4ff.], dass derartige Softwaresysteme auf der Basis eines Referenzsystems standardisierbar waren. Anhand des Modells eines manuell bedienten Lagers beschrieb er typische Funktionen, die durch WMS standardmäßig unterstützt werden mussten [tHom99, S. 15ff.]. Der Entwicklung standardisierter WMS in den letzten Jahren folgend, wurden die Funktionen und unterstützten Prozesse standardisierter WMS deutlich umfangreicher. Trotz der vielfältigen Ausprägungen von WMS existierten jedoch nach wie vor Standardabläufe in logistischen Systemen [tHoS03, S. 23]. Im Umkehrschluss bedeutete dies, dass der Leistungsumfang eines WMS Rückschlüsse auf standardmäßige Lagerprozesse zuließ. 
Verschiedene nationale und internationale Unternehmen erstellten und veröffentlichten Marktstudien über den Leistungsumfang von WMS. Mit der „Internationalen Marktstudie WMS“ des Fraunhofer IML stand die im Vergleich umfangreichste Datenbasis zur Verfügung. Die Marktstudie beinhaltete die Leistungsprofile 65 eigenständiger, standardisierter WMS (Stand 03.01.2006). Da die erfassten WMS eigenständig waren und in zahlreichen Unternehmensklassen branchenübergreifend eingesetzt wurden, war die Datenbasis als repräsentativ für den Leistungsumfang von Standardsoftware in der Domäne „Intralogistik“ anzusehen. Die Datenbasis beinhaltete ca. 1.220 Merkmale und Ausprägungen in Bezug auf Funktionen beziehungsweise von der Software unterstützte Prozesse. So ergab sich eine Datenbasis, die 79.300 (=1.220 Merkmale x 65 WMS) Informationen enthielt. Diese umfassende Datenbasis wurde genutzt, um ein Referenzmodell für die vergleichende Evaluation zu entwickeln. Das hierauf beruhende Referenzmodell war nach der Definition von Fettke/Loos [FeLo04, S. 18] ein Masterreferenzmodell, da es verschiedene Unternehmensklassen beinhaltete.

Ziel der Evaluation war es nachzuweisen, dass das RPM „Automotive Ersatzteillogistik“ eine Teilmenge des Masterreferenzmodells und somit ebenfalls repräsentativ im Sinne eines Referenzmodells war. Die analytische, theoriegeleitete Evaluation des RPM erfolgte in drei Phasen, um die Referenzmodelle unter strukturellen und inhaltlichen Gesichtspunkten zu bewerten. Das Vorgehen ist in der folgenden Abbildung dargestellt:

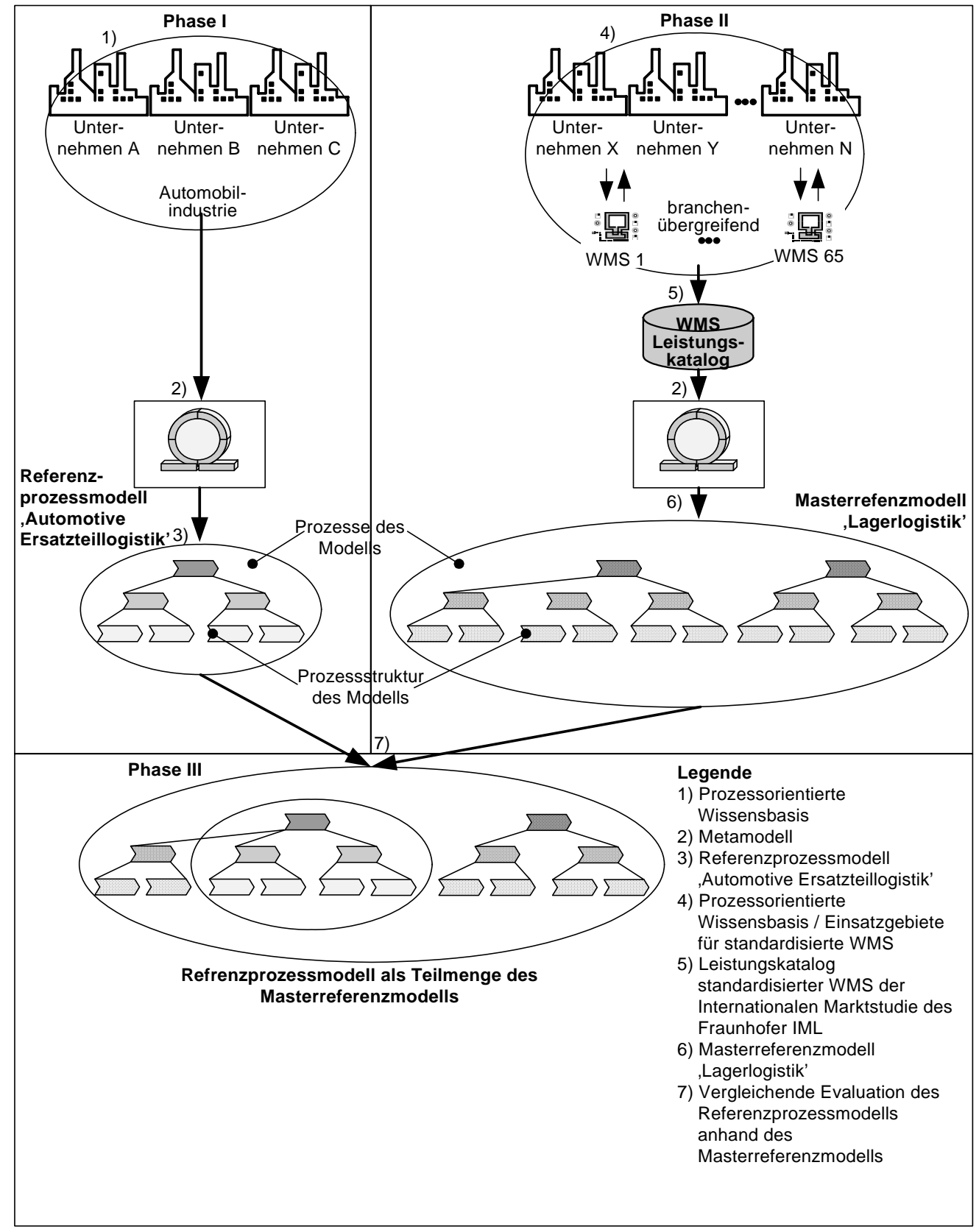

Abbildung 4: Stellung der Evaluation im Kontext der Arbeit 


\section{Phase I - Evaluationsobjekt}

Ausgangspunkt war die Überlegung, ein branchenspezifisches RPM aus einer prozessorientierten Wissensbasis abzuleiten. Hierzu wurde das $\Omega$-Metamodell entwickelt, dessen Durchlaufen im Rahmen einer Fallstudie zu dem Evaluationsobjekt RPM „Automotive Ersatzteillogistik“ führte.

\section{Phase II - Strukturelle Evaluation}

Der strukturelle Vergleich der beiden Referenzmodelle (das RPM „Automotive Ersatzteillogistik“ und das Masterreferenzmodell) orientierte sich an der dreistufigen Hierarchie des RPM „Automotive Ersatzteillogistik“. Es wurde überprüft, ob das Masterreferenzmodell ebenfalls drei Hierarchiestufen aufwies. Weiterhin wurde evaluiert, ob die zwei ordnenden Hierarchiestufen „Hautprozesse“ und „Prozessmodule“ äquivalente Hierarchiestufen in dem Masterreferenzmodell besaßen.

\section{Phase III - Inhaltliche Evaluation}

Die inhaltliche Übereinstimmung der beiden Referenzmodelle erfolgte anhand einer Zuordnung der Prozesselemente des RPM „Automotive Ersatzteillogistik“ zu denen des Masterreferenzmodells. Der Grad der Übereinstimmung war ein Maß für die inhaltliche Äquivalenz der beiden Prozessmodelle.

\section{Ergebnis}

Im Ergebnis war das RPM „Automotive Ersatzteillogistik“ eine nahezu 100\%ige Teilmenge des Masterreferenzmodells. Beide Modelle wiesen drei Hierarchiestufen auf, $98 \%$ aller Prozesse konnten eindeutig beziehungsweise logisch dem Masterreferenzmodell zugeordnet werden. Die einschlägigen Logistikprozesse wurden vollständig von dem RPM abgedeckt. Mit der Evaluation des Referenzmodells wurde die Relevanz des Modells wissenschaftlich nachgewiesen. Fettke/Loos stellten fest, dass mit der Evaluierung anhand eines Masterreferenzmodells untersucht wird, ,whether the facts represented in a reference model are equivalent to the representation in the master reference model." [FeLo03, S. 85]. Der Anspruch des RPM, repräsentativ für die Prozesse in der Domäne „Automobile Ersatzteillogistik“ zu sein, war durch die analytische Evaluation anhand eines Masterreferenzmodells erfüllt, da dieses als repräsentativ für die Domäne „Lagerlogistik“ angesehen werden musste. Die Struktur und der Inhalt des RPM fanden in den wesentlichen Ausprägungen Äquivalente in dem Masterreferenzmodell.

\section{Schlussbetrachtung}

\subsection{Erkenntnisse}

\section{Metamodel für die Referenzmodellierung}

Für die systematische Transformation von implizitem Prozesswissen in explizites wurde ein sechsphasiges Metamodell entwickelt. Die Evaluation bestätigte, dass das $\Omega$-Metamodell ein zweckdienliches Vorgehen für die Erstellung von RPM war, da nach ihm das - als repräsentativ evaluierte - RPM „Automotive Ersatzteillogistik“ entwickelt werden konnte.

\section{Detailliertes Referenzprozessmodell „Automotive Ersatzteillogistik“}

Durch die Anwendung des $\Omega$-Metamodells im Rahmen einer Fallstudie wurden die Logistikprozesse im Untersuchungsraum einer empirischen Generalisierung unterzogen und in das RPM „Automotive Ersatzteillogistik“ überführt. Das Modell bestand aus 509 ,atomaren“ Prozesselementen, die zu 52 Prozessmodulen und acht Hauptprozessen hierarchisch zusammengefasst wurden. $98 \%$ der Prozesse konnten direkt beziehungsweise logisch dem Masterreferenzmodell zugeordnet werden. Der Detaillierungsgrad erreichte einen Wert von 5,5 bezogen auf den Ordnungsrahmen, ein derart feines Referenzprozessmodell wurde bislang in der Wissenschaft nicht diskutiert. Das vorhandene Prozesswissen wurde erheblich erweitert und detailliert.

Das entwickelte Modell war vollständig in Bezug auf die logistischen Abläufe. Die Relevanz des Modells wurde in der wissenschaftlich fundierten Evaluation nachgewiesen. Als Teilmenge eines repräsentativen Masterreferenzmodells erhob es für sich ebenfalls den Anspruch eines repräsentativen Modells in seiner Domäne. Ein empirischer Nachweis der Vollständigkeit und Folgerichtigkeit des Modells wurde so erbracht. Mit dem unternehmensübergreifenden Referenzprozessmodell wurde die wissenschaftliche Grundlage für die unternehmensspezifische Prozessstandardisierung in der Domäne „Automotive Ersatzteillogistik“ gelegt.

\section{Grundlegender Nachweis der generischen Erstellung von Prozessketten}

Für die Verwaltung der Prozessmodelle wurde eine Prozess-Workbench konzipiert und prototypisch realisiert. Mit ihr wurde der Forderung nach der generischen Erstellung von Prozessketten nachgekommen, um der Dynamik im Geschäftsprozessmanagement zu begegnen. Ein prototypischer Nachweis der Wandelbarkeit von Prozessketten auf der Basis ,atomarer“ Prozesselemente wurde erbracht. 
Wissenschaftlich fundierter Ordnungsrahmen für Referenzprozessmodelle in der Intralogistik Aus den einschlägigen Logistikprozessen wurde erstmalig ein Ordnungsrahmen für Prozesse im Lager abgeleitet. Er begrenzt die Domäne „Lagerlogistik“. Mit ihm wurde die Vergleichbarkeit logistischer Prozessmodelle ermöglicht: Qualitativ in Bezug auf die Vollständigkeit der Prozesse und quantitativ im Hinblick auf den Detaillierungs- bzw. Abstraktionsgrad.

\subsection{Ausblick}

Im Rahmen des Transferprojekts ist die sich direkt anschließende Aufgabe die Erstellung weiterer, branchenspezifischer Referenzprozesse, um vielfältige, über die Automobilindustrie hinausgehende, logistische Aufträge abdecken zu können und die Prozesskompetenz nachzuweisen. Hierzu dient zum einen das $\Omega$-Metamodell als geeignete Methode und zum anderen das entwickelte RPM „Automotive Ersatzteillogistik“, aus dessen „atomaren“ Prozesselementen weitere Referenzmodelle abgeleitet werden können. Im Rahmen der Entwicklung eines Geschäftsmodells für das „Virtuelle Lager“ erfolgt die Entwicklung eines Algorithmus für die betriebswirtschaftliche Leistungsverrechnung auf Basis der bereits definierten Referenzprozesse. Aus Sicht der Informationstechnik werden die Ergebnisse der vorliegenden Arbeit für ein prototypisches Konzept zur Softwareunterstützung unter Nutzung logistischer Referenzprozesse genutzt. Hierunter fällt das Bestandsmanagement („Virtuelle Bestandsführung“) ebenso wie die Disposition der logistischen Ressourcen (Lager- und Transportkapazitäten).

Die „Internationale Marktstudie WMS“ des Fraunhofer IML beinhaltet eine einmalige, repräsentative Datenbasis hinsichtlich des Leistungsumfangs standardisierter Systeme. Die Weiterentwicklung der Prozess-Workbench und deren anschließende Integration in die Tools der Marktstudie führen sicherlich zu einer erheblichen Effizienzsteigerung bei der prozessorientierten Auswahl standardisierter WMS.

Mit der Entwicklung des Masterreferenzmodells wurde die enge Verbindung von Prozessen und Funktionen in standardisierten Informationssystemen untermauert. Das entwickelte Referenzprozessmodell kann als Basis für die Entwicklung eines WMS herangezogen werden. Der Detaillierungsgrad erlaubt ein Verständnis der komplexen Abläufe in einem Zentrallager.

Die Kenntnis über Prozesse ist die Grundlage

- für das Prozessdesign und die Prozessstandardisierung in der Planungsphase,

- für die Auswahl von Software zur Unterstützung der Operationen und

- für die prozessorientierte Entwicklung von Software.

Der letzte Punkt betont, dass Softwareentwicklung einen Überblick über die zu unterstützenden Prozesse verlangt und interdisziplinär erfolgen sollte. Hier schlägt das vorliegende RPM eine Brücke, wenn auch „nur“ für eine Domäne.

Diese verschiedenen Sichten auf einen Prozess knüpfen an das Paradigma von Smith/Fingar der „third wave of business process management" [SmFi02, S. 13] an, das die multifunktionale Nutzung eines Prozessmodells postuliert. Das Referenzprozessmodell für die Domäne „Automotive Ersatzteillogistik“ ist in Kombination mit der Prozess-Workbench ein Beitrag zur Prozessstandardisierung in der Intralogistik.

\section{Literatur}

[BeSc04]

[FeLo04]

[FeLo03]

[Figg05]

[Figg06]

[Schü98]
Becker, Jörg; Schütte, Reinhard: Handelsinformationssysteme. 2. Auflage. Frankfurt am Main: Verlag moderne industrie, 2004

Fettke, Peter; Loos, Peter: Systematische Erhebung von Referenzmodellen - Ergebnisse der Voruntersuchung (Paper 29). In: Working Papers of the Research Group Information Systems \& Management, Lehrstuhl für Wirtschaftsinformatik und BWL, Johannes Gutenberg Universität, Mainz, 2004

Fettke, Peter; Loos, Peter: Multiperspective Evaluation of Reference Models - Towards a Framework. In: Jeusfeld; M. A.; Pastor, Ó. (Eds.): Conceptual Modeling for Novel Application Domains - ER 2003 Workshops ECOMO, IWCMQ, AOIS, and XSDM, Chicago, IL, USA, October 13, 2003. Lecture Notes in Computer Science (LNCS), Vol. 2814, Springer, Berlin et al. 2003, S. 80-91.

Figgener, Olaf: Den Prozessgedanken unterstützen. IN: F+H „Fördern und Heben“Sonderausgabe Lager Marktbild 2005, 55 (2005), 1, S. 75-77

Figgener, Olaf: Standards für die Intralogistik. IN: Software in der Logistik - MarktSpiegel. München: Huss-Verlag, 2006, S. 26-29

Schütte, Reinhard: Grundsätze ordnungsmäßiger Referenzmodellierung. Konstruktion und anpassungsorientierte Modelle. Wiesbaden: Gabler Verlag, 1998 
[SFB06]

[SmFi02]

[tHHe06]

[tHom99]

[tHoS03]

[VDA04]

[VDI04]

[Wild01]
N.N.: Sonderforschungsbereich 559 - Modellierung großer Netze in der Logistik. Universität Dortmund (http://www.sfb559.uni-dortmund.de/, Aufruf am 19.04.2007) Smith, Howard; Fingar, Peter: Business Process Management - The Third Wave. Tampa, FL: Megha-Kiffer Press, 2002 ten Hompel, Michael; Heidenblut, Volker: Taschenlexikon Logistik - Abkürzungen, Defintionen und Erläuterungen der wichtigsten Begriffe aus Materialfluss und Logistik. Berlin [u.a.]: Springer, 2006

ten Hompel, Michael: Lagerverwaltungssysteme. GamBit GmbH, Dortmund, 1999 ten Hompel, Michael; Schmidt, Thorsten: Warehouse Management. Berlin [u.a.]Springer, 2003

N.N.: Future Automotive Industry Structure (FAST) 2005 - die neue Arbeitsteilung in der Automobilindustrie. Frankfurt am Main: Verband der Automobilindustrie VDA, 2004

N.N.: Operative Logistikkennzahlen von Wareneingang bis Versand. In: VDIGesellschaft Fördertechnik Materialfluss Logistik (Hrsg.): VDI-Richtlinie: VDI 4490 (Entwurf). Berlin: Beuth Verlag, 2004

Wildemann, Horst: Logistik Prozessmanagement. München: TCW TransferCentrum-Verlag, 2001 